\title{
Copépodos planctónicos de la Bahía de Chetumal, Caribe Mexicano: variaciones estacionales durante un ciclo anual
}

\author{
Planktonic copepods of Chetumal Bay, Mexican Caribbean: \\ seasonal variations during an annual cycle \\ Cuauhtémoc Ruíz-Pineda ${ }^{1}$, Eduardo Suárez-Morales ${ }^{1, *}$ \\ y Rebeca Gasca ${ }^{1}$
}

${ }^{1}$ El Colegio de la Frontera Sur, Unidad Chetumal, A.P. 424, Chetumal, Quintana Roo 77014, México. *esuarez@ecosur.mx

\begin{abstract}
Planktonic copepods are representative members of the coastal and estuarine biota. In Chetumal Bay, western Caribbean, the relevance of monitoring the copepod community at different scales is widely recognized. Zooplankton was sampled monthly during 3 seasons (northerlies, rainy, dry) during a year cycle (1997). The composition and distribution of planktonic copepods and their monthly and seasonal abundance was analyzed to assess the influence of environmental parameters on this community. Each seasonal period was characterized by distinct relative abundance values of the most abundant species. Acartia tonsa was dominant during the 3 seasons, with high relative abundances year-round (67-92\%). The ANOSIM (Bray Curtis similarity analysis) showed significant differences of the community among seasons thus confirming a seasonal dynamics; a monthly significant variability was also observed. The PCA (Principal Components Analysis) showed that temperature and salinity explained $82 \%$ of the observed variability; additional analyses indicated that salinity best explains the distributional patterns of the species. Two distinct groups were detected, one representing the resident estuarine community with high abundances of $A$. tonsa, low abundances of A. lilljeborgi, low species richness, and sites of the inner areas of the bay and of nortes (January and November). The second group represents a foreigner coastal-neritic community with low abundances of $A$. tonsa, presence of A. lilljeborgi, high diversity of species; it includes sites of the dry and rainy seasons plus those of the external zone of the bay. The local dominance of $A$. tonsa in Chetumal is explained by the low concentration of predators and the low densities of A. lilljeborgi, mostly restricted to areas with higher salinity and also by its probable positive correlation with oxygen concentration which probably limits its populations in a hypoxic system like Chetumal.
\end{abstract}

Key words: Estuaries, zooplankton, copepods, Acartia tonsa, western Caribbean

Resumen.- Los copépodos del plancton son representativos de la biota de sistemas estuarino-costeros. En la Bahía de Chetumal, Caribe de México, se reconoce la importancia de su estudio y monitoreo en distintas escalas. Se obtuvieron muestras de zooplancton durante las épocas climáticas de nortes, lluvias y secas de un ciclo anual (1997). Se determinó la composición, distribución y abundancia mensual y estacional de los copépodos para determinar la influencia de algunas variables ambientales sobre esta comunidad. Cada época mostró distintos valores relativos de abundancia de las especies más abundantes. Acartia tonsa fue dominante en las tres épocas, con altos valores de abundancia relativa (67-92\%) en todo el ciclo anual. El análisis de ANOSIM (Análisis de Similitud Bray Curtis) mostró diferencias significativas de la comunidad entre épocas confirmando una dinámica estacional; también se observó una variabilidad significativa a escala mensual. El Análisis de Componentes Principales mostró que la temperatura y la salinidad explicaron el $82 \%$ de la variabilidad observada y análisis adicionales indicaron que la salinidad explica mejor los patrones de distribución de las especies. EI ANOSIM y el SIMPER (Porcentaje de Similitud) revelaron dos grupos distintos, uno que representa la comunidad residente con altas abundancias de A. tonsa, bajas abundancias de $A$. lilljeborgi, baja riqueza de especies; incluye localidades de la zona interna de la bahía y los meses de nortes (enero y noviembre). El segundo grupo correspondió a una comunidad foránea nerítico-costera, con baja abundancia de A. tonsa, presencia de A. lilljeborgi, elevada diversidad de especies y estaciones de secas y lluvias además de las estaciones externas de la bahía. La dominancia local de A. tonsa en Chetumal se explica por la baja abundancia de depredadores y las bajas densidades de A. lilljeborgi, que está principalmente restringida a áreas con mayor salinidad y también a su probable correlación positiva con el oxígeno disuelto, lo que posiblemente limita sus poblaciones en un sistema hipóxico como Chetumal.

Palabras clave: Estuarios, zooplancton, Acartia tonsa, copépodos, Caribe occidental 


\section{INTRODUCCIÓN}

Los copépodos son microcrustáceos que predominan en el zooplancton de los sistemas estuarino-costeros (Escamilla et al. 2011) y son los más importantes consumidores primarios en estos ambientes (Calbet et al. 2000). Su diversidad es relativamente baja en las zonas costeras o estuarinas donde está dominada por formas que se han adaptado a los cambios de salinidad que caracterizan a estos ambientes (SuárezMorales 1994, Vásquez-Yeomans et al. 2012). El seguimiento de estos y otros sistemas costeros a largo plazo es una herramienta esencial para detectar cambios en la estructura y diversidad de su biota en distintas escalas (Cruz-Motta et al. 2010). Por estas razones el estudio de los patrones de variabilidad de los copépodos en las comunidades acuáticas estuarinas podría revelar información importante de la dinámica de la comunidad local del zooplancton, con implicaciones a otros sistemas estuarino-costeros tropicales. En general, el monitoreo de la comunidad del zooplancton permite detectar y dar seguimiento a niveles de perturbación y deterioro ecológico (Branco et al. 2007).

En los sistemas costero-estuarinos del sur del Golfo de México y Caribe noroccidental los copépodos han sido estudiados pero los trabajos han sido aislados o limitados a ciertos sistemas (Suárez-Morales 2003). Se ha destacado su importancia regional como indicadores de condiciones ambientales a partir de sus patrones de distribución y abundancia, principalmente en el caso de especies de Acartia (Escamilla et al. 2001), representativas en estos sistemas (Ordóñez-López \& Ornelas-Roa 2003, Escamilla et al. 2011). También se ha mostrado que son sensibles a cambios estacionales en sistemas costeros de la costa Caribe de México (Suárez-Morales \& Gasca 1996). Estos elementos justifican ampliamente el estudio de las poblaciones de copépodos planctónicos en distintas escalas (Suárez-Morales \& Gasca 1996, Álvarez-Cadena \& Segura-Puertas 1997, ÁlvarezCadena et al. 2007).

La Bahía de Chetumal es un extenso sistema estuarino situado en la costa oriental de la Península de Yucatán, México; la bahía recibe aportes freáticos de diversos orígenes, pero principalmente del Río Hondo, que marca una frontera natural con Belice. La bahía es una zona de Conservación Ecológica, Santuario del Manatí (Morales-Vela et al. 2000) y ha sido reconocida como área de crianza de larvas de peces (Carrillo et al. 2009). En términos ecológicos es un sistema oligotrófico con zonas y episodios de hipoxia derivados del creciente aporte de desechos agrícolas en su zona más interna (Izasa et al. 2006). La influencia marina al interior de la bahía es limitada y variable, su salinidad es de baja a moderada (8-18). Desde el punto de vista climatológico, la región muestra una dinámica estacional marcada por las épocas de secas, lluvias y nortes (Carrillo et al. 2009).

Los primeros estudios respecto a los copépodos del plancton de la bahía de Chetumal se efectuaron a partir de muestras recolectadas en 1990 y 1991 (Suárez-Morales 1994, 1995) y revelan la importancia local de Acartia tonsa y A. lilljeborgi como especies que coexisten en estos sistemas y cuyas variaciones deben ser evaluadas a lo largo del tiempo para detectar cambios en la comunidad. Los patrones de distribución de estas 2 especies se han asociado a cambios hidrográficos incluyendo gradientes de salinidad (ÁlvarezCadena \& Cortés-Altamirano 1990, Suárez-Morales 1994, Lopes et al. 1998) y ritmos de marea (Escamilla et al. 2001).

El objetivo del presente estudio fue describir y analizar los patrones de la composición, abundancia y distribución de la comunidad local de copépodos, a partir de muestras de zooplancton obtenidas en la Bahía de Chetumal, México durante el ciclo anual 1997, y determinar la respuesta a la variación de factores ambientales. Los resultados fueron analizados en función de la estructura de la comunidad y sus variaciones espacio-temporales.

\section{Materiales Y MÉTODOS}

Se obtuvieron 78 muestras de zooplancton en 2 meses representativos de cada una de las 3 épocas climáticas de la región durante 1997: nortes (enero y noviembre), secas (marzo y mayo) y lluvias (julio y septiembre). Se efectuaron arrastres circulares superficiales (uno por sitio) con una duración de 10 min en 13 estaciones distribuidas en toda la bahía (Fig. 1). Se utilizó una red de plancton de 0,45 m de diámetro y abertura de malla de $100 \mu \mathrm{m}$. El volumen de agua filtrada se estimó con un flujómetro digital General Oceanics adaptado a la boca de la red. Los valores de volumen filtrado obtenidos durante los muestreos fluctuaron entre 132 y $232 \mathrm{~m}^{3}$. Las muestras se fijaron y preservaron en formalina al $4 \%$ neutralizada con borato de sodio. Simultáneamente, se tomaron datos de salinidad por medio de un refractómetro Aquafarma, de temperatura superficial del agua $\left({ }^{\circ} \mathrm{C}\right)$ con un termómetro de cubeta y con una sonda se estimó la profundidad (m) del sitio de obtención de la muestra. La identificación de las especies de copépodos se realizó con la ayuda de claves de identificación regionales (Campos-Hernández \& Suárez-Morales 1994, SuárezMorales 1995).

Se utilizaron, de acuerdo con la biomasa de cada muestra obtenida, alícuotas de 1/2, 1/4 y 1/8 para la cuantificación de los copépodos. Considerando el volumen de agua filtrada por la red, se estandarizó la abundancia total y la de cada especie a 


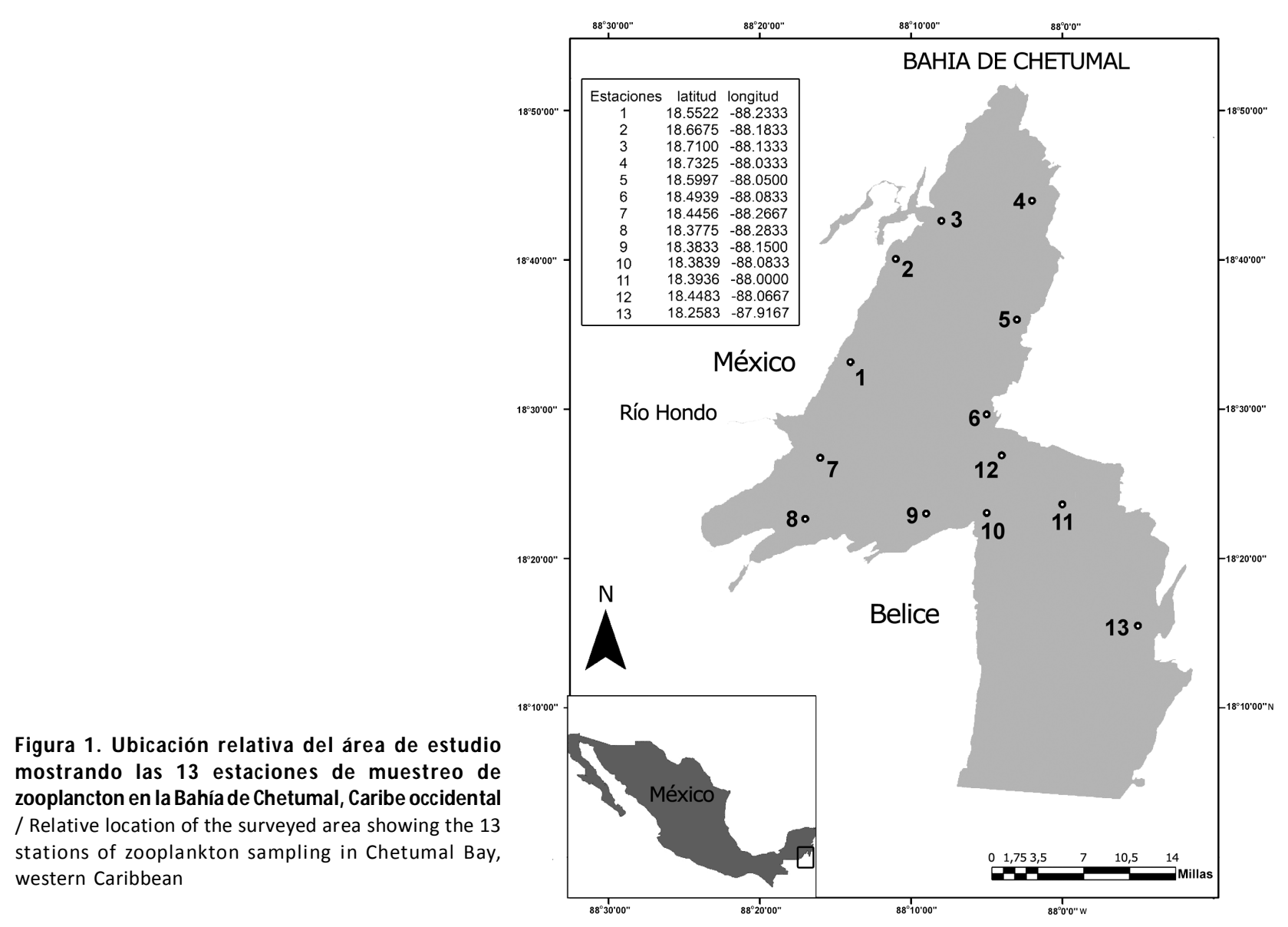

ind. $1000 \mathrm{~m}^{-3}$. Los valores de abundancia por especie se presentan como promedio y para su análisis fueron transformados a $\log _{10}(x+1)$ para obtener un conjunto de datos con distribución normal y homoscedástica (Legendre \& Legendre 1983). Se calcularon también las medias geométricas (MG) de las abundancias mensuales y de cada época con sus intervalos de confianza (IC) (Sokal \& Rolf 1995). Los valores de biomasa de zooplancton obtenidos en el área de estudio por Vásquez-Yeomans et al. (2012) para el ciclo 1997, con el mismo equipo de colecta, fueron utilizados para determinar su relación con la comunidad local de copépodos.

La semejanza entre las muestras (abundancia por especie) se determinó mediante un análisis de similitud (ANOSIM) con el índice de Bray-Curtis utilizando un enlace promedio de grupo como medida de distancia y se aplicó una prueba de significancia SIMPROF (Perfil de Similitud) para los grupos obtenidos. Los resultados se graficaron en un Escalamiento Multidimensional No Métrico (NMDS). Adicionalmente, se efectuaron Análisis de Similitud (ANOSIM) para contrastar las $\mathrm{H}_{0}$ de que no existen diferencias de los parámetros de la comunidad entre los meses, las épocas o los sitios de muestreo. Se calcularon y analizaron los porcentajes de similitud SIMPER para conocer las diferencias en los grupos analizados (meses, sitios, épocas y los resultantes en el NMDS) y determinar las especies que tuvieron una mayor contribución a la similitud entre sitios de cada uno de los grupos. Se estimó el índice de diversidad de Shannon para todas las muestras (Shannon 1948).

Los datos ambientales de normalizaron y se analizó su distribución espacial y temporal en los sitios muestreados mediante un ACP. Para seleccionar la combinación de variables que mejor concuerdan con los patrones de distribución de las especies, se realizó un análisis de correlación de Spearman entre la matriz de variables ambientales basadas en Distancias Euclidianas y el patrón de similitud de Bray-Curtis de las especies usando la rutina BEST. Se realizó una superposición de las abundancias de las especies (ind. $1000 \mathrm{~m}^{-3}$ ) con el gráfico de ACP para visualizar su distribución y su relación con algunas de las variables estimadas. Los análisis estadísticos se efectuaron con ayuda del software PRIMER 6 (Plymouth Routines in Multivariate Ecological Research) (Clarke \& Warwick 2001). 
Se elaboraron los mapas de distribución de temperatura, salinidad, abundancia y diversidad con el método de interpolación utilizando el programa ArcGis 10.2.1.(ESRI) ${ }^{1}$

\section{Resultados}

\section{Temperatura Y SALINIDAD}

Nortes. Durante la época de nortes las temperaturas más elevadas se encontraron en las zonas media e interna de la bahía (est. 1-4, 7,10) y los registros más bajos se observaron en la zona externa de la bahía (Fig. 2A). Las salinidades más elevadas se observaron en las estaciones 10-13 y las menores en las estaciones 1,7 y 8 , cerca de la desembocadura del Río Hondo (Fig. 3A).

Secas. Durante esta temporada las estaciones de la zona interna de la bahía presentaron los valores de más elevados de temperatura (est. 1-5) (Fig. 2B). Las estaciones externas 10, 12 y 13 presentaron los registros de salinidad más elevados y la zona norte del sistema los más bajos (Fig. 3B).

Lluvias. En esta temporada las temperaturas más altas ocurrieron en las estaciones 1-3, 5, 6 (Fig. 2C) y las mayores salinidades en la zona externa (est. 10-13) (Fig. 3C).

Durante el ciclo anual los registros de temperatura más elevados ocurrieron en la zona interna de la bahía (est. 1-5). Las estaciones ubicadas en la zona externa de la bahía (10-13) tuvieron los registros de salinidad más elevados (Figs. 2D, 3D).

\section{CoMPOSICIÓN Y ABUNDANCIA}

Durante el ciclo anual se identificaron 8 especies de copépodos planctónicos en el área de estudio: Acartia tonsa Dana, 1849, A. lilljeborgi Giesbrecht, 1888, Tortanus angularis Ohtsuka, 1992, Ditrichocorycaeus amazonicus (Dahl, 1894), Calanopia americana Dahl, 1894, Paracalanus quasimodo Bowman, 1971 y Temora stylifera (Dana, 1849); se determinó su abundancia mensual y por época. Acartia tonsa representó el $88 \%$ de abundancia relativa anual en la comunidad local de copépodos, seguida por Tortanus angularis (8\%), A. lilljeborgi $(3 \%)$ y el $1 \%$ restante estuvo integrado por $D$.

Tabla 1. Variaciones mensuales de la abundancia total de copépodos (ind. $1000 \mathrm{~m}^{-3}$ ) en la Bahía de Chetumal, México durante el periodo de estudio. Los valores se representan con medias geométricas (MG) e intervalos de confianza (IC) / Monthly variations of total copepod abundance (ind. $1000 \mathrm{~m}^{-3}$ ) in Chetumal Bay, Mexico during the surveyed period. Values are presented as geometrical means (MG) with Confidence Intervals $(\mathrm{Cl})$

\begin{tabular}{cccccc}
\hline \multicolumn{1}{c}{ Enero } & \multicolumn{1}{c}{ Marzo } & \multicolumn{1}{c}{ Mayo } & \multicolumn{1}{c}{ Julio } & Septiembre & Noviembre \\
\hline 74726 & 28301 & 21868 & 55198 & 25727 & 190234 \\
$\mathrm{MG}=79,29$ & $\mathrm{MG}=123,89$ & $\mathrm{MG}=102,15$ & $\mathrm{MG}=214,75$ & $\mathrm{MG}=64$ & $\mathrm{MG}=494$ \\
$\mathrm{IC}=29-216$ & $\mathrm{IC}=55-277$ & $\mathrm{IC}=47-221$ & $\mathrm{IC}=90-510$ & $\mathrm{IC}=29-143$ & $\mathrm{IC}=168-1451$ \\
\hline
\end{tabular}

${ }^{1}$ Z<http://www.esri.com/arcgis $>$ amazonicus, C. americana, L. scotti, P. quasimodo y $T$. stylifera (Fig. 5).

La abundancia total de copépodos de la bahía tuvo variaciones mensuales. La mayor abundancia se observó durante noviembre, seguida por enero y julio mientras que los menores valores ocurrieron en mayo (Tabla 1).

Variación estacional (nortes). Las mayores abundancias totales de copépodos se presentaron en la zona cercana a la desembocadura del Río Hondo y en la estación 10; las menores se observaron en la zona nororiental de la bahía (Fig. 4A). La media de la abundancia durante esta época fue la mayor con respecto a las otras 2 épocas (Tabla 2). Durante esta época Acartia tonsa fue la especie más abundante; representó más del $90 \%$ del total de la comunidad de copépodos local durante esta temporada, seguida por T. angularis con 7\% (Tabla 2, Fig. 5A).

\section{Abundancia estacional (secas)}

En esta temporada se observaron las mayores abundancias totales de copépodos en la zona más interna, adyacente a Río Hondo (est. 1, 8, 7) y las menores hacia la zona más externa de la bahía y en las estaciones 2 y 3 (Fig. 4B). La abundancia durante secas fue la más baja entre las épocas (Tabla 2). Durante esta temporada dominaron 3 especies: A. tonsa, A. lilljeborgi y $T$. angularis. La primera tuvo sus menores valores relativos y las 2 siguientes especies tuvieron sus mayores abundancias relativas en el ciclo anual: 10 y 20\%, respectivamente (Tabla 2, Fig. 5B).

\section{Abundancia estacional (lluvias)}

La zona cercana a la desembocadura del Río Hondo tuvo los promedios de abundancia más altos; los menores valores se observaron en el sector oriental y externo del sistema (Fig. 4C), tendencia observada también en el comportamiento anualizado de la abundancia local de copépodos (Fig. 4D). La abundancia durante esta época fue intermedia entre nortes y secas (Tabla 2). Al igual que se observó en nortes, $A$. tons a fue altamente

Tabla 2. Variacionesestacionales de la abundancia total de copépodos (ind. $1000 \mathrm{~m}^{-3}$ ) y abundancia relativa de Acartia tonsa (\%) en la Bahía de Chetumal, M éxico durante el periodo de estudio. Los valores se representan con medias geométricas (MG) y sus intervalos de confianza (IC) / Seasonal variations of total copepod abundance (ind. $1000 \mathrm{~m}^{-3}$ ) and relative abundance of Acartia tonsa (\%) in Chetumal Bay, Mexico during the surveyed period. Values are presented as geometrical means (MG) with their Confidence Intervals (CI)

\begin{tabular}{lccc}
\hline Abundancia & Nortes & Secas & Lluvias \\
\hline Total de copépodos & 264969 & 50169 & 80925 \\
& $\mathrm{MG}=102, \mathrm{IC}=49-212$ & $\mathrm{MG}=113, \mathrm{IC}=64-197$ & $\mathrm{MG}=80, \mathrm{IC}=45-141$ \\
Acartia tonsa & 91 & 67 & 92 \\
\hline
\end{tabular}



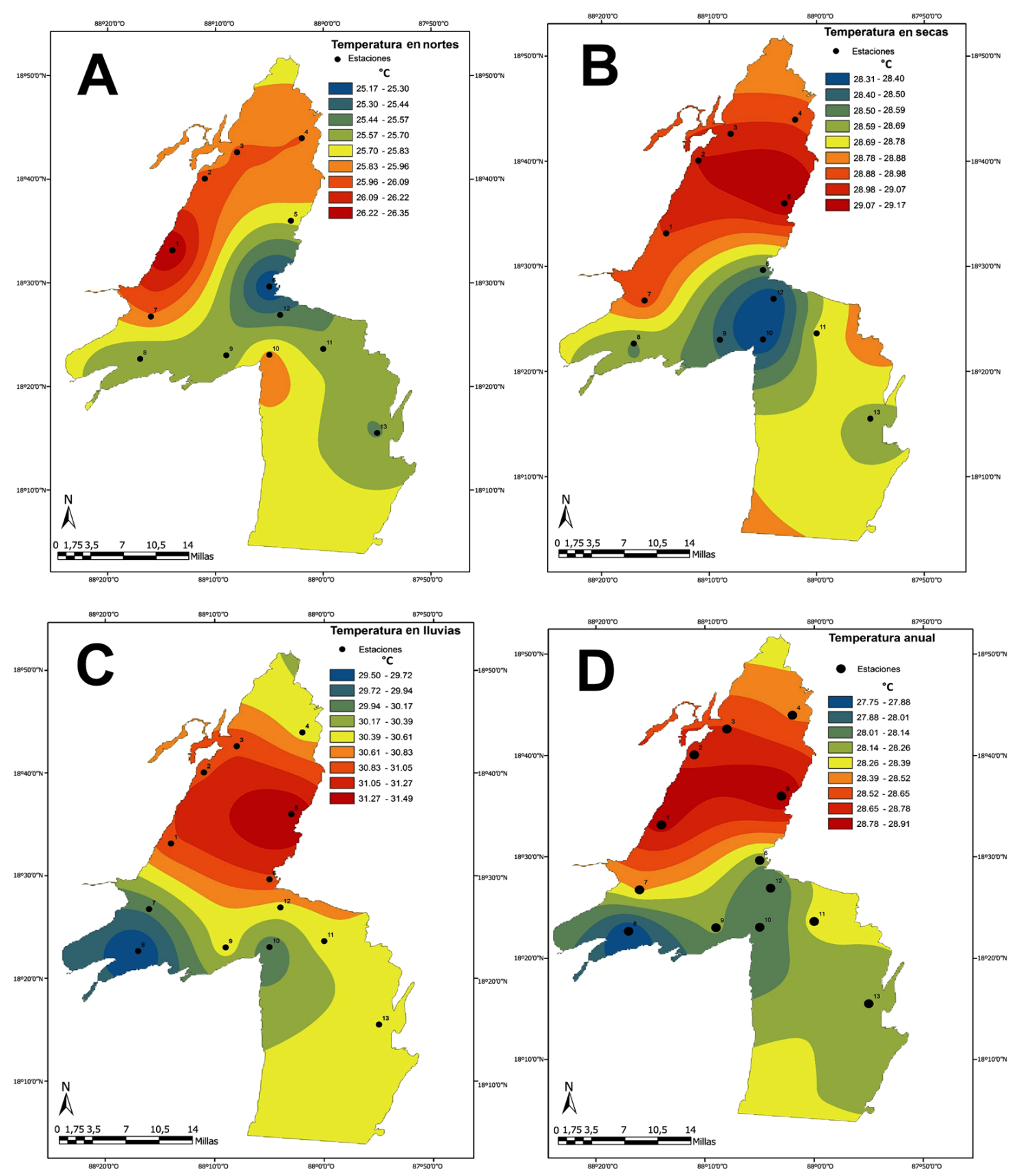

Figura 2. Variación por época y distribución de la temperatura superficial $\left({ }^{\circ} \mathrm{C}\right)$ en la Bahía de Chetumal durante 1997. A) nortes; B) secas; C) lluvias; D) distribución anual / Seasonal variation and distribution of the surface temperature $\left({ }^{\circ} \mathrm{C}\right)$ in Chetumal Bay during 1997 . A) nortes season; B) dry season; C) rainy season; D) annual distribution 

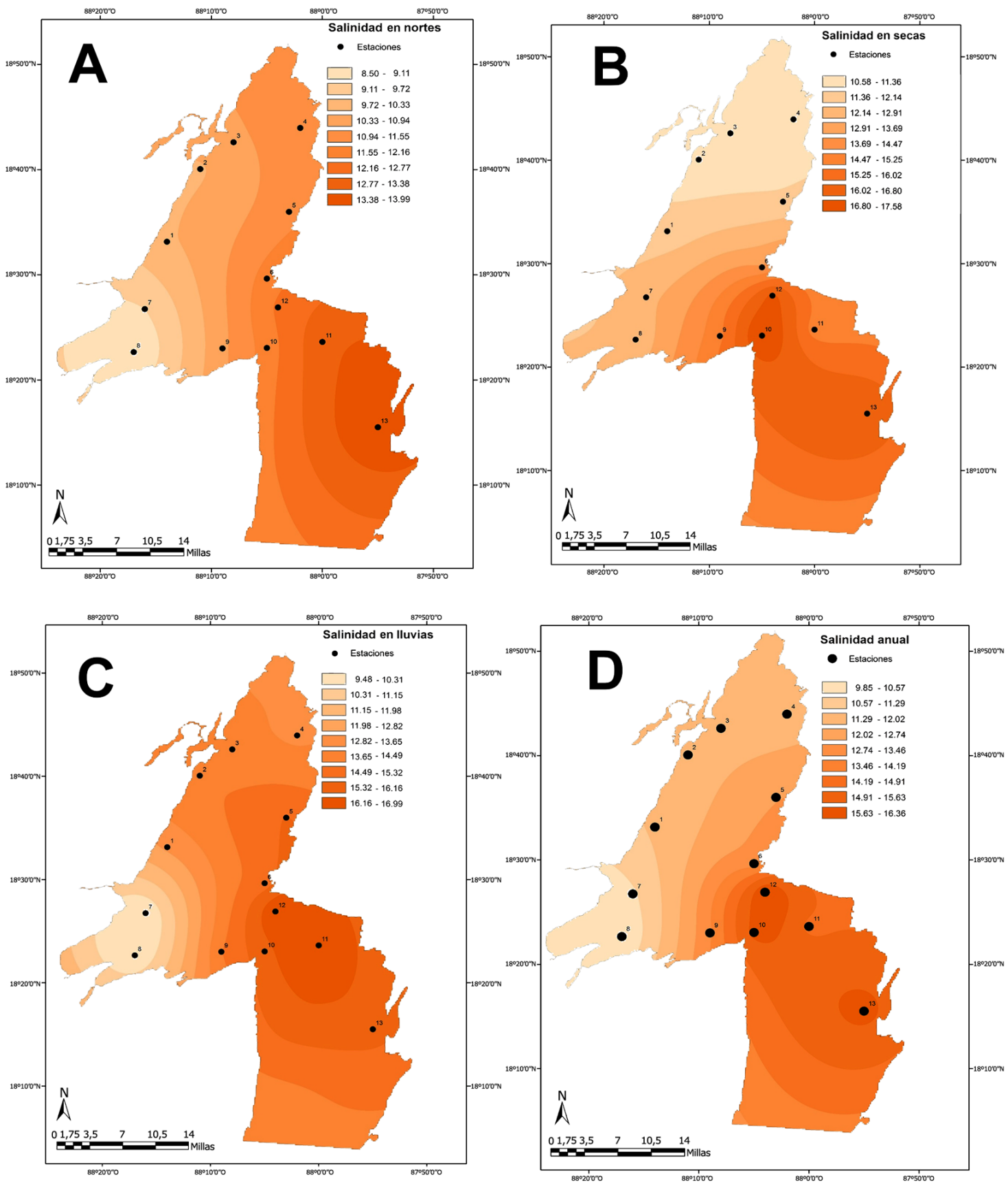

Figura 3. Variación por época y distribución de la salinidad superficial en la Bahía de Chetumal durante 1997. A) nortes; B) secas; C) lluvias; D) distribución anual / Seasonal variation and distribution of the surface salinity in Chetumal Bay during 1997. A) nortes season; B) dry season; C) rainy season; D) annual distribution

Ruíz-Pineda et al.

Copépodos planctónicos en un estuario del Caribe occidental 

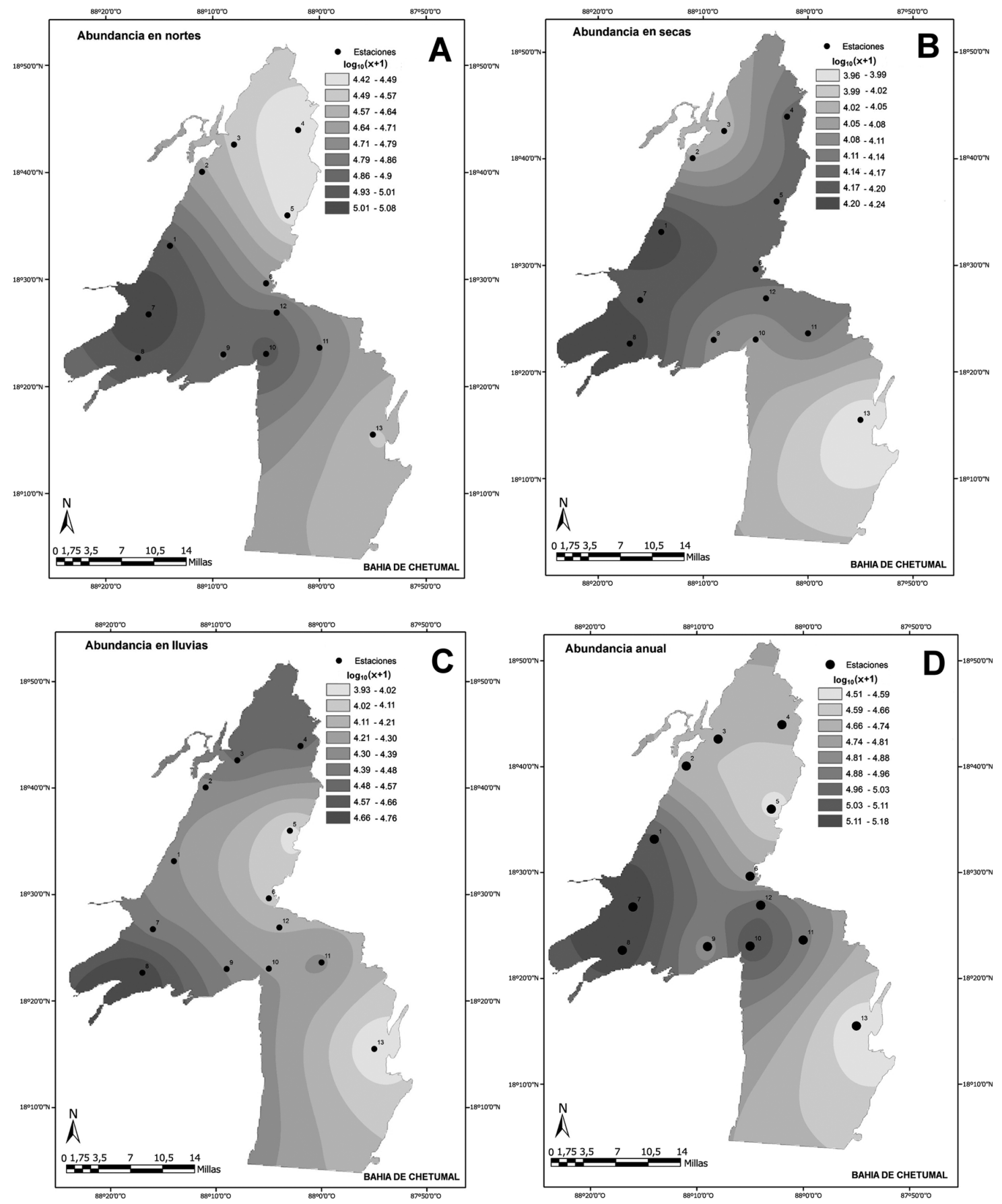

Figura 4. Variación por época y distribución de la abundancia total de copépodos (ind. $1000 \mathrm{~m}^{-3}$ ) en la Bahía de Chetumal durante 1997. A) nortes; B) secas; C) lluvias; D) distribución anual / Seasonal variation and distribution of the total abundance (ind. $1000 \mathrm{~m}^{-3}$ ) of copepods in Chetumal Bay during 1997. A) nortes season; B) dry season; C) rainy season; D) annual distribution 

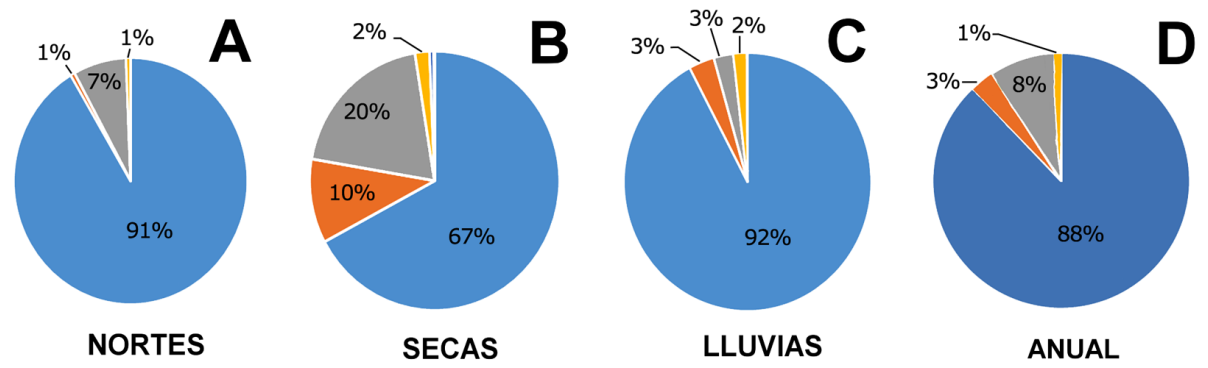

$\begin{array}{ll}\text { Acartia tonsa } & \text { Tortanus angularis } \\ \text { Acartia lilljeborgi } & \text { Ditrichocorycaeus amazonicus }\end{array}$

Figura 5. Abundancia relativa por época y anual de las especies más abundantes de copépodos en la Bahía de Chetumal durante 1997. A) nortes; B) secas; C) lluvias; D) distribución porcentual anual / Relative abundance (seasonal and annual) of the most abundant species of copepods in Chetumal Bay during 1997. A) nortes; B) dry; C) rainy; D) annual percentual distribution

dominante, seguida por A. lilljeborgi y T. angularis, ambas con una abundancia relativa de $3 \%$ (Tabla 2, Fig. 5C).

\section{AnÁlisis de la COMUNIDAD}

A partir del ACP se obtuvo que $81,7 \%$ de la variabilidad ambiental se relaciona con 2 factores: salinidad y temperatura. La correlación resultante de la rutina BEST entre las matrices ambiental y biológica indicó que la variable que mejor explica el patrón de distribución de las especies de copépodos fue la salinidad $(\pi=0,48, P<0,001)$. En la figura 6 se muestra la relación entre la abundancia de cada especie en todas las localidades con los parámetros ambientales obtenidos a partir del ACP. Acartia tonsa mostró una distribución poco afectada por la temperatura o la salinidad y fue abundante en todos los meses pero con menores valores en enero; también tendió a aparecer más abundantemente en condiciones menos salinas (Fig. 6A). En contraste, A. lilljeborgi fue más abundante hacia las zonas más salinas y con mayor temperatura; sus menores abundancias se presentaron en enero y en las localidades internas en noviembre (Fig. 6B). Tortanus angularis tuvo sus mayores abundancias hacia las zonas y meses con menor temperatura y salinidades medias a bajas (Fig. 6C). Ditrichocorycaeus amazonicus, al igual que A. lilljeborgi tendió a ser más abundante en meses y zonas con mayor temperatura y salinidad (Fig. 6D). Calanopia americana y $P$. quasimodo tuvieron una clara tendencia a ser más abundantes en zonas y meses con mayor salinidad y temperatura (Figs. 6E y F).

\section{Diversidad de Shannon}

En general, en el periodo estudiado se obtuvieron bajos valores de diversidad de Shannon. Las estaciones más cercanas a la boca de la bahía (10-13) mantuvieron los valores más altos de este índice durante el ciclo anual. En la temporada de nortes las estaciones 1 y 2 fueron las menos diversas y la 13, en la zona externa de la bahía, fue la más diversa (Fig. 7A). Durante secas la mayor diversidad se registró en la zona externa de la bahía y la menor en la zona media (Fig. 7B). En lluvias la menor diversidad se encontró en la zona interna de la bahía, aumentando hacia la zona externa (Fig. 7C). El comportamiento anual fue en general homogéneo pero con tendencia a mostrar valores más elevados hacia la zona más externa de la bahía (Fig. 7D).

\section{Similitud de Bray-Curtis y NMDS}

El análisis de similitud de Bray-Curtis y el NMDS generado a partir de estos datos reveló la agrupación de muestras similares en cuanto a la composición de especies y su abundancia en cada una de ellas. Se obtuvo un patrón representado por 2 grupos a una similitud de $72,1 \%(\pi=1,1, P<0,001)$. El grupo 'a' (Fig. 8) fue el más numeroso y estuvo formado por sitios de la época de nortes (enero y noviembre) más los de la zona interna de la bahía de otros meses. El análisis SIMPER indicó que $A$. tonsa es altamente relevante para la conformación del grupo (47,8\%), seguida por T. angularis (35,2\%); el grupo sólo incluyó 4 especies y excluyó a A. lilljeborgi. El grupo 'b' tuvo un menor número de sitios pero integró las localidades con mayor diversidad de Shannon y mayor riqueza de especies; se formó principalmente por estaciones de marzo más las estaciones externas de la bahía (9-13). Acartia tonsa y $T$. angularis contribuyeron 34,9 y $22 \%$ al grupo, respectivamente; A. lilljeborgi representó sólo el 19,8\% y estuvo ausente del grupo 'a'. Según los resultados de este análisis esta última 
A

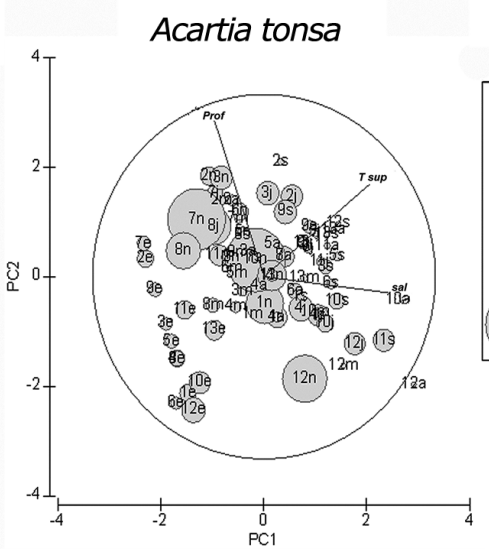

C

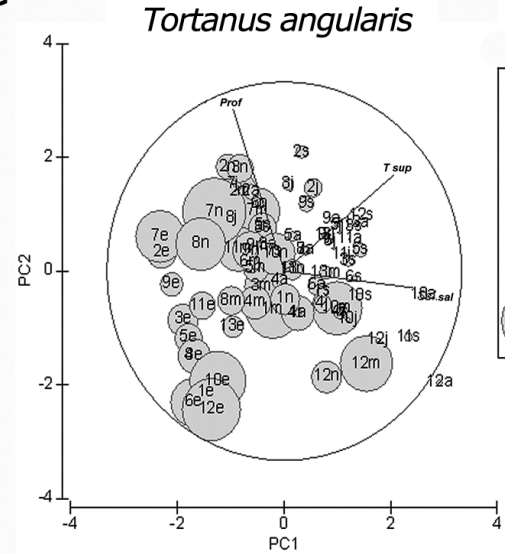

$\mathrm{E}$

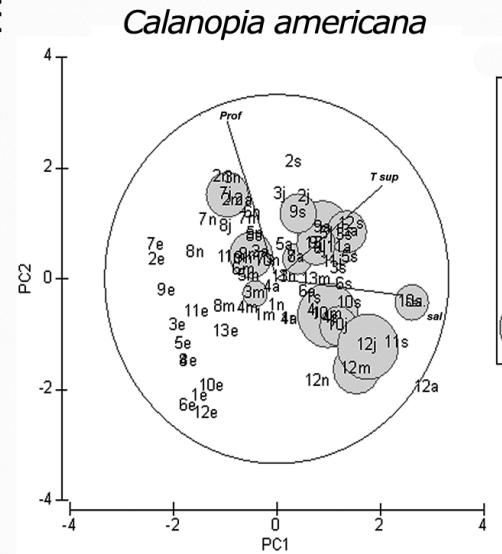

B
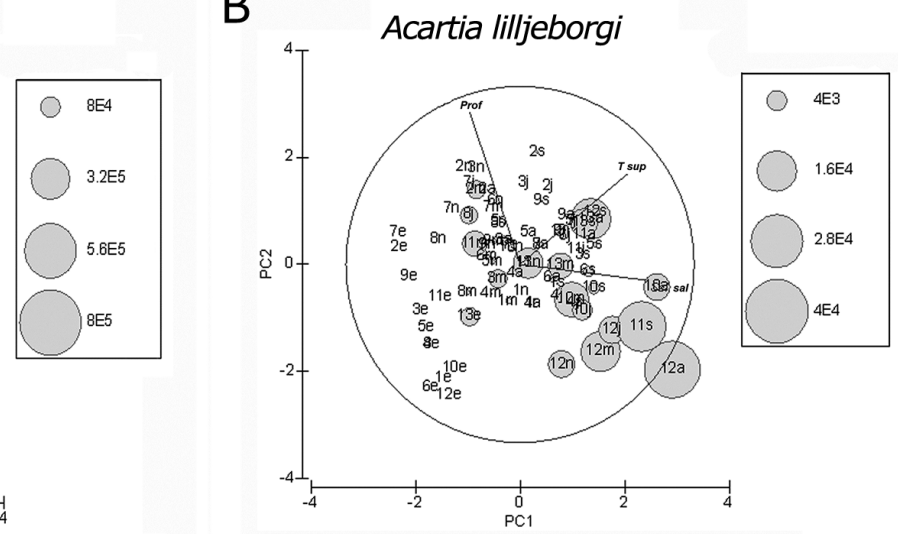

D

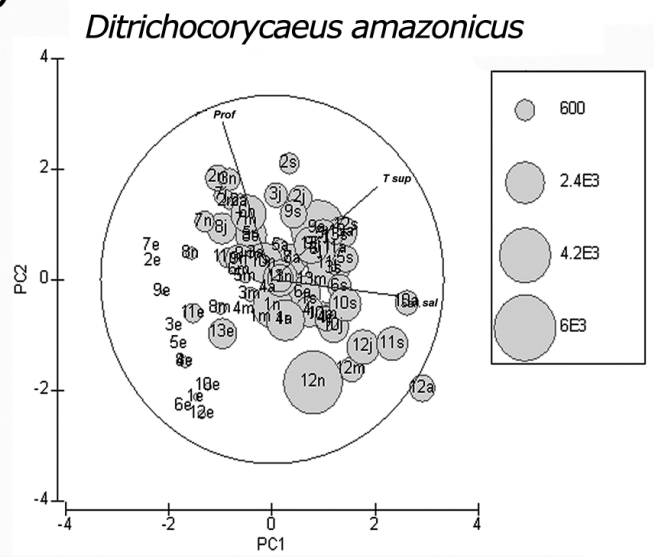

$\mathrm{F}$

Paracalanus quasimodo

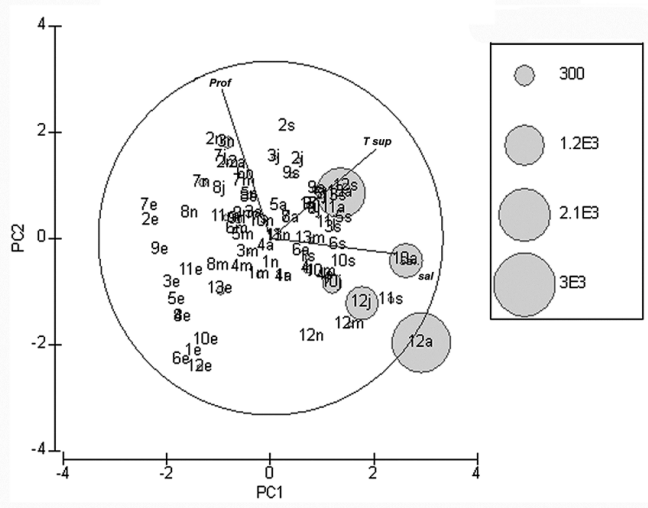

Figura 6A-F. Representación de la abundancia de las 6 especies de copépodos más comunes en las muestras analizadas con los ejes resultantes del ACP sobrepuestos. Escala en ind. $1000 \mathrm{~m}^{-3}$. Prof. $=$ profundidad, Tsup=temperatura superficial, sal=salinidad, $e=e n e r o, n=n o v i e m b r e, m=m a r z o, a=$ mayo, $\mathbf{j}=$ julio, $\mathbf{s}=$ septiembre/ Representation of the abundance of the 6 commonest species of copepods in the analyzed samples with the superimposed axes resulting from the ACP. Scale in ind. $1000 \mathrm{~m}^{-3}$. Prof= depth, Tsup= surface temperature, sal= salinity, e= January, $n=$ November, $m=$ March, $a=$ May, j= July, s= September 

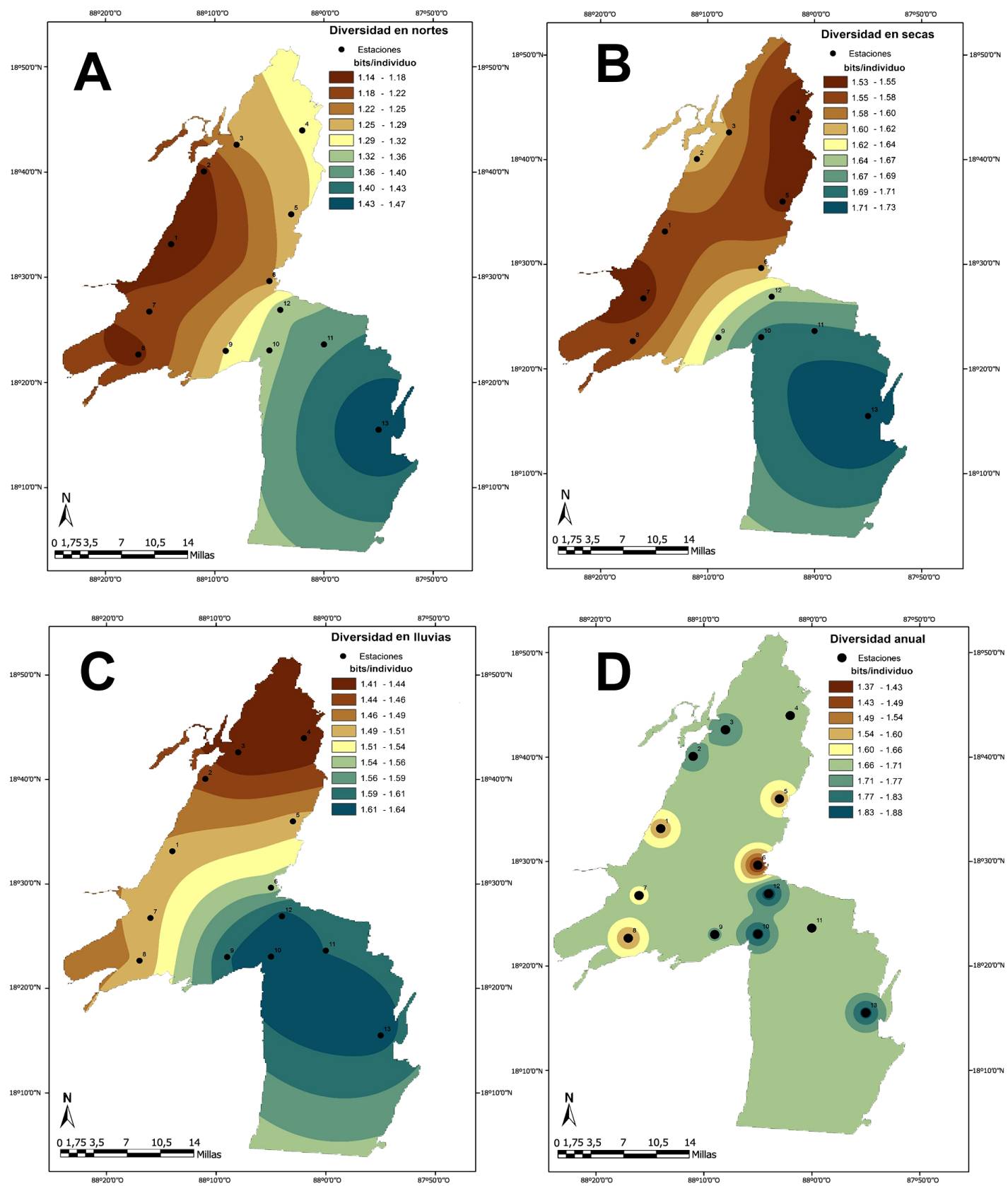

Figura 7. Variaciones por época y distribución de la Diversidad de Shannon en la Bahía de Chetumal durante 1997. A) nortes; B) secas; C) lluvias; D) distribución anual / Seasonal variations and distribution of Shannon's Diversity values in Chetumal Bay during 1997. A) nortes; B) dry; C) rainy; D) annual distribution 


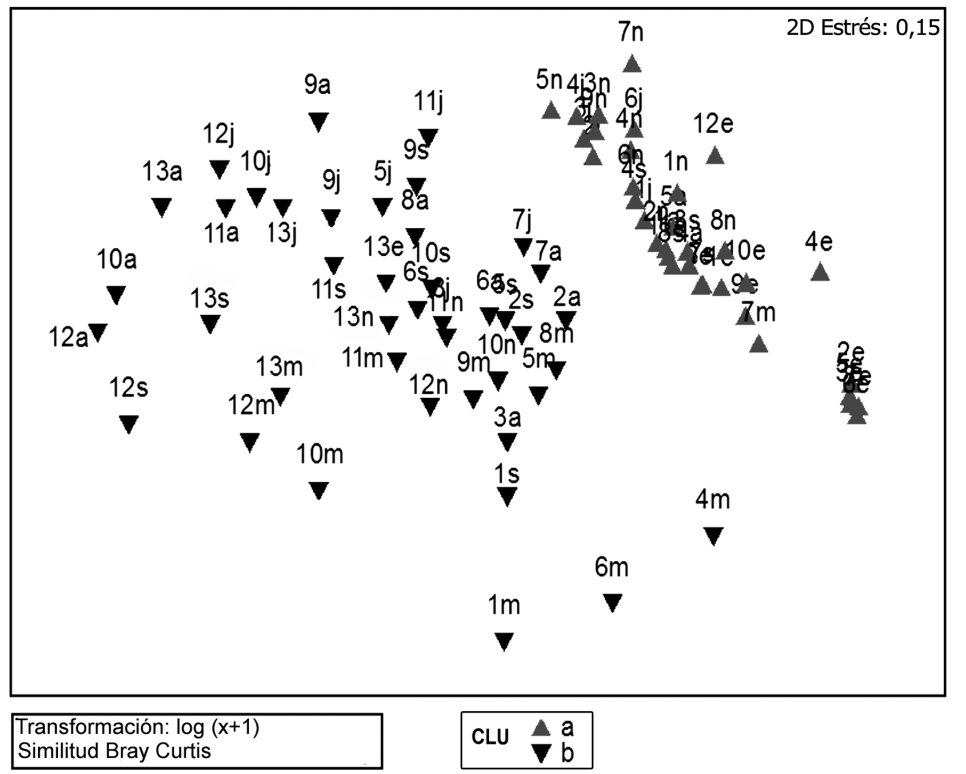

Figura 8. Resultado del análisis de similitud de Bray Curtis (ANOSIM) y su representación en un NM DS; se muestra el arreglo en 2 grupos de muestras (a y b) / Results of the Bray Curtis Similarity analysis and its NMDS representation; the arrangement in 2 groups ( $a$ and $b$ ) is shown

especie es la que más contribuye a diferenciar ambos grupos (40\%), además de Ditrichocorycaeus amazonicus (15\%) y Tortanus angularis (13\%).

El ANOSIM indicó que las $\mathrm{H}_{0}$ se rechazan pues se obtuvieron diferencias significativas entre épocas $(\mathrm{R}=0,201, P$ $<0,001)$, meses $(\mathrm{R}=0,207, P<0,001)$ y sitios de muestreo $(\mathrm{R}=0,128, P<0,001)$. Hubo algunos meses de épocas distintas que fueron similares, como mayo y septiembre $(\mathrm{R}=65.9, P<$ 0,001) y mayo y julio $(\mathrm{R}=42,7, P<0,001)$, pero julio y septiembre, representativos de lluvias, fueron parecidos entre sí $(\mathrm{R}=36,90, P<0,001)$. Estas afinidades se atribuyen a periodos de transición o a condiciones climáticas similares en distintas épocas. Enero, representativo de la época de nortes fue muy distinto a los demás meses $(\mathrm{R}=0,403-0,517, P<$ $0,001)$, lo que explica parte de las diferencias que distinguieron a esta época. En cuanto a las localidades se observó que los sitios 10-13 son similares $(\mathrm{R}<-0,08, P<0,001)$ al igual que los sitios 1-6 ( $<<0,45, P<0,001)$, lo que apoya el patrón espacial observado en la comunidad local de copépodos.

El SIMPER reveló que los mayores porcentajes de similitud se registraron entre las estaciones internas $(1,4,6$ y 7) y las más externas $(11,12$ y 13) con porcentajes de disimilitud mayores a 30\%. Las estaciones con una similitud mayor al $72 \%$ fueron 1, 4, 8 y 7 en la zona interna, con amplia dominancia de A. tonsa; en la zona externa las estaciones 11 y 13 fueron las más similares, con la mayor diversidad, las menores abundancias de A. tonsa y la presencia de A. lilljeborgi, T. angularis y $P$. quasimodo.

\section{Discusión}

La bahía de Chetumal reúne atributos de un sistema costero semi-cerrado por su escasa profundidad, la reducida extensión de su boca, su baja energía interna, su oligotrofia y la débil influencia marina, limitada a la zona externa que difiere del resto de la bahía (Gasca et al. 1994, Carrillo et al. 2009). Las mayores salinidades de las zonas externas del sistema en todas las épocas de muestreo y en el promedio anual confirmaron esta sectorización. Estas condiciones favorecen cierta homogeneidad general en los parámetros físicos, químicos y biológicos al interior de la bahía y su biota zooplanctónica local es representativa de estas condiciones y en general es uniforme en cuanto a composición respecta, con una baja riqueza de especies (Gasca \& Castellanos 1993), tal como se observó en la comunidad de copépodos analizada. Sin embargo, los análisis efectuados evidenciaron cambios temporales y espaciales detectables en esta comunidad. Como se presenta en otros sistemas estuarinos tropicales (Escamilla et al. 2001, 2011; Costa et al. 2011), las variaciones de salinidad es el factor más relevante en la formación del perfil de la comunidad local de copépodos, siendo determinante en su distribución espacial y composición lo que permite la formación de grupos de especies característicos de zonas o condiciones particulares en la bahía. 


\section{Abundancia total y DIVERSIDAD}

En un estudio previo en la bahía (Suárez-Morales 1994), la mayor abundancia media de copépodos fue de 1146 ind 1000 $\mathrm{m}^{-3}$. En 1997 se observó una media considerablemente mayor: 38545 ind. $1000 \mathrm{~m}^{-3}$; estas diferencias muestran variaciones interanuales importantes en la comunidad local de copépodos. Estos cambios pueden atribuirse a factores como las fluctuaciones en el régimen de lluvias o a la influencia antropogénica. Los continuos incrementos en el aporte de aguas residuales agrícolas y/o urbanas en la bahía inducen la eutroficación de este sistema básicamente oligotrófico con un importante incremento de fitoplancton (Herrera-Silveira et al. 2009); esto podría explicar la mayor abundancia de copépodos en 1997 respecto a 1991. Acartia tonsa, la especie más abundante y representativa del sistema es un eficiente consumidor de fitoplancton (Tester \& Turner 1988, Turner \& Tester 1989) y sus poblaciones se pueden incrementar rápidamente en la presencia de alimento abundante (Teixeira et al. 2010). Adicionalmente, es previsible que la apertura de un canal de comunicación con el frente marino, efectuada en 2006, haya provocado cambios recientes en la comunidad local de copépodos pues además de incrementos en la salinidad, se ha documentado un importante proceso de eutroficación en la bahía que fue detectado en 2000 (Herrera-Silveira et al. 2009) pero posiblemente inició antes. Es por esto que los datos aquí presentados adquieren una mayor importancia para evaluar las variaciones interanuales de la comunidad del zooplancton a mediano plazo y entender la dinámica de este sistema costero.

Las especies de Acartia son formas típicamente eurihalinas y usualmente predominan en los sistemas estuarinos tropicales pero suelen estar acompañadas de otras especies menos dominantes (Escamilla et al. 2001, 2011, Ordóñez-López \& Ornelas-Roa 2003, Álvarez-Cadena et al. 2007, Araujo et al. 2008) o incluso ser desplazadas como especies secundarias en sistemas costeros con mayor influencia marina (Álvarez-Silva et al. 2006, Costa et al. 2011). La escasa influencia de aguas marinas en la bahía de Chetumal limita el número de especies al interior del sistema según lo muestra la distribución del índice de Shannon durante este estudio, cuyos valores más altos resultaron en las zonas más externas de la bahía (estaciones 913). Considerando trabajos previos (Suárez-Morales 1994 , 1995) y los resultados del presente análisis, en la bahía coexisten tan sólo 8-10 especies de copépodos planctónicos. La riqueza de especies es baja en relación con la de otros sistemas estuarino-costeros de la región. Por ejemplo, en la bahía Ascensión, en la costa caribeña de México, Suárez-Morales \& Gasca (1996) reportaron 36 especies; en Celestún, Yucatán, México, Ordóñez-López \& Ornelas-Roa (2003) observaron 17 especies y en el complejo lagunar de Chelem se registraron
13 especies (Escamilla et al. 2011). Estas diferencias se atribuyen a las peculiares condiciones fisiográficas (i.e., somera, oligotrófica, con escasa influencia marina, baja energía interna) de la bahía de Chetumal que favorecen que la comunidad residente de copépodos esté compuesta básicamente por un reducido grupo de especies eurihalinas con afinidades estuarinocosteras.

\section{VARIABILIDAD TEMPORAL}

A partir de los resultados del ANOSIM se comprobaron diferencias significativas entre las épocas, lo que apoya la idea de estacionalidad en la dinámica temporal de la comunidad de copépodos. Adicionalmente, las diferencias mensuales también fueron significativas, lo que sugiere que aún dentro de cada época existen variaciones detectables, por lo que se considera que a pesar de la baja energía propia de la bahía y de considerarse una zona hipóxica (Díaz \& Rosenberg 2008), se trata de un sistema cuya comunidad de copépodos tiene una variabilidad más amplia de lo que podría esperarse.

Otro indiciode la estacionalidad de esta comunidad se percibió en la proporción de abundancia de copépodos por época con respecto al total anual; durante lluvias representó $24 \%$ del total, secas $15 \%$ y en nortes $61 \%$ del total anual. Así, durante esta última época las mayores abundancias del ciclo anual coincidieron con los promedios de salinidad más bajos cuyos gradientes locales definen la distribución de la comunidad y fueron más marcados en la época de nortes. Las precipitaciones asociadas con esta temporada podrían haber incrementado el aporte de nutrientes hacia la bahía (Álvarez-Legorreta 2009) y explicar parcialmente la elevada abundancia de copépodos durante esta época. Es claro que otros factores como las épocas reproductivas de las especies o la disponibilidad de alimento deben ser considerados en este rubro. En la bahía de la Ascensión, un sistema adyacente pero con características fisiográficas distintas a Chetumal, los copépodos fueron también más abundantes en nortes (Suárez-Morales \& Gasca 1996). El hallazgo de Temora stylifera se considera un indicio de que aguas marinas ingresan ocasionalmente a los sectores más internos por flujos costeros (Carrillo, com. pers. $)^{2}$ pues se trata de una especie de afinidad nerítico-oceánica (Bowman 1971, Turner 1984). Se debe considerar que esta especie no había sido encontrada previamente en la bahía y su registró fue en la parte más interna de la bahía (est. 4) y durante la época de nortes, caracterizada por generar zonas de mezcla de la biota del plancton (Suárez-Morales \& Gasca 1996, Suárez-Morales et al. 1997). Por tanto, la presencia de esta especie en una zona tan interna y de baja salinidad podría ser resultado de estos procesos locales y también de la tendencia a ser más abundante en la época invernal (Bowman 1971). 
La temporada de lluvias se caracterizó por una marcada disminución de la salinidad en la zona externa de la bahía como resultado de las precipitaciones, lo cual limitó la distribución de especies marinas y favoreció la proliferación de A. tonsa, que como forma eurihalina residente, representó el $92 \%$ de la comunidad local de copépodos (Fig. 5C). El comportamiento de esta comunidad durante lluvias fue semejante al observado en secas pues se mantuvo un gradiente marcado por la separación de las estaciones con mayor influencia marina y se observó mezcla de la comunidad residente que ocupó el resto de la bahía. En bahía Ascensión la separación entre lluvias y secas es más definida (Suárez-Morales \& Gasca 1996). Durante secas se observó la mayor riqueza de especies, lo que coincidió con las mayores salinidades en la zona externa de la bahía, lo que indica una mayor influencia del frente marino y ello se refleja en una mayor representación de especies costeromarinas en el sistema y una elevada diversidad, un patrón que se ha observado en otros sistemas costeros tropicales (Araujo et al. 2008). A este efecto se asocia la presencia local de $P$. quasimodo, C. americana y L. scotti, propias de aguas costeras de mayor salinidad (González \& Bowman 1965, Bowman 1971, Campos-Hernández \& Suárez-Morales 1994, Suárez-Morales \& Gasca 1996).

\section{VARIABILIDAD ESPACIAL}

Durante el ciclo anual estudiado, la comunidad de copépodos se distribuye en la bahía de Chetumal siguiendo un patrón que incluye 2 zonas que marcan, en el caso del grupo «a», la comunidad residente, propia de las zonas media e interna de la bahía, caracterizada por las mayores densidades de $A$. tonsa, baja representación de $A$. lilljeborgi y baja diversidad de especies. La agrupación «b» definió una comunidad temporal, restringida a las localidades más externas de la bahía, la baja abundancia de A. tonsa, la presencia de A. lilljeborgi y la mayor diversidad de especies en el sistema. Esto coincide con lo encontrado por Escamilla et al. (2011) en otro sistema lagunar del Golfo de México, en donde las áreas y épocas de dominancia de estas dos especies de Acartia fueron, en gran medida, mutuamente excluyentes.

\section{Biomasa}

La biomasa (peso húmedo) de zooplancton varió espacial y temporalmente en 1997 (Vásquez-Yeomans et al. 2012). En la época de nortes se obtuvieron los valores más altos, seguida por secas y lluvias. La abundancia de copépodos y la biomasa del zooplancton presentaron valores elevados en las zonas adyacentes a la desembocadura del Río Hondo en las distintas épocas (Fig. 4, Vásquez-Yeomans et al. 2012). Esta tendencia

${ }^{2}$ El Colegio de la Frontera Sur (ECOSUR) Chetumal, México coincide con lo observado por Herrera-Silveira et al. (2009) en cuanto a una mayor productividad primaria cerca de la desembocadura del Río Hondo. En la estación 10 también ocurrieron abundancias de copépodos notoriamente elevadas. En la zona en que su ubica esa estación, Ortíz-Hernández \& Sáenz-Morales (1999) y Álvarez-Legorreta (2009) observaron el tamaño de grano más fino en sedimentos y mayores concentraciones de hidrocarburos aromáticos policíclicos HAP y materia orgánica. Estos datos sugieren que esta área de la bahía representa un reservorio de sedimentos finos (limos, arcillas) y materia orgánica; se especula que podría favorecer una alta productividad local y explicar los altos valores de biomasa y abundancia de copépodos observados en este punto.

\section{Acartia tonsa y A. lilljeborgi}

La población de A. tonsa representó un $88 \%$ del total de copépodos encontrados en la bahía; esta acentuada dominancia permite detectar cambios temporales en la estructura de la comunidad mediante el seguimiento de una sola especie. Este copépodo tiene una amplia tolerancia a valores cambiantes de salinidad, una alta resiliencia aún en condiciones de alta turbidez y bajas concentraciones de clorofila (Derisio et al. 2014) y suele ser dominante en las zonas más internas de los sistemas costeros (Sabatini 1989) incluyendo ambientes hipersalinos (Britton \& Morton 1989) y aguas oligohalinas como la bahía de Chetumal (Suárez-Morales 1994). Esta capacidad se refleja en los resultados obtenidos en esta bahía pues no se observaron correlaciones significativas entre las variables ambientales y la abundancia de la especie (ACP) aunque sus mayores abundancias se asociaron con salinidades medias y bajas (Fig. 6A). Lo anterior sugiere que las variaciones locales de salinidad no limitan la abundancia local de $A$. tonsa, por lo que otros factores como la disponibilidad de alimento, las tasas reproductivas de la propia especie y la depredación pueden jugar un papel importante en la dinámica de esta especie. Esto coincide con los resultados de Calliari et al. (2006), quienes demostraron que las salinidades moderadas a bajas favorecen el éxito reproductivo de $A$. tons $a$, especie que por su baja tasa de reconversión alimento/biomasa se debe alimentar más y por mayor tiempo, haciéndola más vulnerable a la depredación por larvas de peces. En la bahía de Chetumal Gasca y Castellanos (1993) encontraron una correlación negativa entre los copépodos y las larvas de peces $(\mathrm{r}=-0,6575, P<0,05)$, que serían los principales depredadores de copépodos en la comunidad del zooplancton. Parte del éxito local de $A$. tonsa se puede relacionar con la baja tolerancia de las larvas y juveniles de peces a soportar las variaciones ambientales (Tester \& Turner 1991, Suárez-Morales 1994), lo que favorecería a esta especie en la comunidad de copépodos (Poulet \& Williams 1991). 
Usualmente A. tonsa y A. lilljeborgi coexisten en estos sistemas estuarino-costeros y para reducir la competencia continua alternan su dominancia ya sea especial o temporalmente (Escamilla et al. 2001, 2011; Sterza \& Fernandes 2006). La amplia y consistente dominancia de $A$. tonsa sobre su congénere en Chetumal, se puede explicar por la preferencia de $A$. lilljeborgi por las zonas de mayor salinidad en la bahía (Fig. 6B), lo que implica una limitación respecto a $A$. tonsa. Por otra parte, es de considerar que $A$. tonsa tiene una tolerancia importante para soportar bajos niveles de oxígeno, de hasta $2,3 \mathrm{ml} \mathrm{L}^{-1}$ (Elliot et al. 2013), por lo que los valores de este factor en Chetumal (5-6,5 $\left.\mathrm{ml} \mathrm{L}^{-1}\right)$ (Herrera-Silveira et al. 2009) no representan limitante alguno para el desarrollo de sus poblaciones. En cambio, se ha encontrado una correlación positiva entre $A$. lilljeborgi y el oxígeno en otro sistema costero del Golfo de México (Escamilla et al. 2011). Este parámetro pudiera explicar en parte la baja abundancia de A. lilljeborgi en la bahía de Chetumal, un sistema catalogado como hipóxico (Diaz \& Rosenberg 2008). Las demás especies muestran intervalos de salinidad con afinidad costero-marina que limitan espacialmente su distribución en la bahía, favoreciendo así la formación de un grupo diverso, poco abundante y con una cobertura limitada a la zona exterior, con mayor influencia marina.

\section{Agradecimientos}

Agradecemos a las personas que nos apoyaron en las tareas asociadas con la preparación de los viajes de muestreo y la obtención de las muestras de zooplancton en la Bahía de Chetumal durante 1997. En particular recibimos el apoyo de Rosa Ma. Hernández, César Quintal, Concepción Mondragón, Soledad Jiménez e Iván Castellanos. Este proyecto fue realizado con el financiamiento del proyecto Zooplancton de El Colegio de la Frontera Sur (ECOSUR) y como parte de la tesis profesional del primer autor (CR-P) en el Instituto Tecnológico de Chetumal (ITCH). Tres revisores anónimos aportaron sugerencias y comentarios constructivos que permitieron mejorar la claridad y presentación de este trabajo; su tiempo y esfuerzo se agradece sinceramente.

\section{LiTERATURA CITADA}

Álvarez-Cadena JN \& R Cortés-Altamirano. 1990. Algunos factores físicos y biológicos que afectan las poblaciones naturales de Acartia tonsa y A. lilljeborgii (Copepoda: Acartiidae) en el estero de Urías, Sinaloa, México. Investigaciones Marinas CICIMAR 5: 69-77.

Álvarez-Cadena JN \& L Segura-Puertas. 1997. Zooplankton variability and copepod species assemblages from a tropical coastal lagoon. Gulf Research Reports 9: 345-355.
Álvarez-Cadena JN, U Ordóñez-López, D Valdés, AR Almaral-Mendívil \& A Uicab-Sabido. 2007. Estudio anual del zooplancton: composición, abundancia, biomasa e hidrología del norte de Quintana Roo, Mar Caribe de México. Revista Mexicana de Biodiversidad 78: 421-430.

Álvarez-Legorreta T. 2009. Contaminación acuática. En: Espinoza-Avalos J, GAIslebe \& HA Hernández-Arana (eds). El sistema ecológico de la bahía de Chetumal / Corozal: costa occidental del Mar Caribe, pp. 205-217. ECOSUR, Chetumal, México.

Álvarez-Silva C, G Miranda, G De Lara \& S Gómez. 2006. Zooplancton de los sistemas estuarinos de Chantuto y Panzacola, Chiapas, en época de secas y lluvias. Hidrobiológica 16: 175-172.

Araujo HMP, DA Nascimento-Vieira, S Neumann-Leitão, R Schwamborn, APO Lucas \& JPH Alves. 2008. Zooplankton community dynamics in relation to the seasonal cycle and nutrient inputs in an urban tropical estuary in Brazil. Brazilian Journal of Biology 68: 751-762.

Bowman TE. 1971. The distribution of calanoid copepods off the southeastern United States between Cape Hatteras and Southern Florida. Smithsonian Contributions to Zoology 96 : $1-64$.

Branco CW, B Kozlowsky-Suzuki \& FA Esteves. 2007. Environmental changes and zooplankton temporal and spatial variation in a disturbed Brazilian coastal lagoon. Brazilian Journal of Biology 67: 251-262.

Britton JC \& B Morton. 1989. Shore ecology of the Gulf of Mexico, 387 pp. University of Texas Press, Austin.

Calbet A, MR Landry \& RD Scheinberg. 2000. Copepod grazing in a subtropical bay: species-specific responses to a midsummer increase in nannoplankton standing stock. Marine Ecology Progress Series 193: 75-84.

Calliari D, CM Andersen, P Thor, E Gorokhova \& P Tiselius. 2006. Salinity modulates the energy balance and reproductive success of co-occurring copepods Acartia tonsa and A. clausi in different ways. Marine Ecology Progress Series 312: 177-188.

Campos-Hernández A \& E Suárez-Morales. 1994. Copépodos pelágicos del Golfo de México y Mar Caribe. Biología y Sistemática, 359 pp. CONACYT/CIQRO, México.

Carrillo L, E Palacios-Hernández, AM Ramírez \& B Morales-Vela. 2009. Características hidrometeorológicas y batimétricas. En: Espinoza-Avalos J, GA Islebe \& HA Hernández-Arana (eds). El sistema ecológico de la bahía de Chetumal / Corozal: costa occidental del Mar Caribe, pp. 12-20. ECOSUR, Chetumal, México.

Castelblanco-Martínez N, J Padilla-Saldívar, H HernándezArana, D Sloane, J Reid \& B Morales-Vela. 2013. Movement patterns of Antillean manatees in Chetumal Bay (Mexico) and coastal Belize: A challenge for regional conservation. Marine Mammal Science 29: 166-182. 
Clarke KR \& RM Warwick. 2001. Change in marine communities: an approach to statistical analysis and interpretation, 172 pp. PRIMER-E, Plymouth.

Cruz-Motta JJ, P Miloslavich, G Palomo, K Iken, B Konar, G Pohle, T Trott, L Benedetti, C Herrera, A Hernández, A Sardi, A Bueno, J Castillo, E Klein, E Guerra, J Gobin, D Gómez, R Riosmena, A Mead, G Bigatti, A Knowlton \& Y Shirayama. 2010. Patterns of spatial variation of assemblages associated with intertidal rocky shores: a global perspective. PLoS ONE 5(12): e14354. <doi:10.1371/journal.pone.0014354>

Costa RM da, P Atique, KG Costa \& LCC Pereira. 2011. Seasonal and spatial variation in hydrological parameters and microzooplankton communities in an Amazonian estuary. Journal of Coastal Research 64: 1477-1481.

Derisio C, M Braverman, E Gaitán, C Hozbor, F Ramírez, J Carreto, F Botto, DA Gagliardini, EM Acha \& H Mianzan. 2014. The turbidity front as a habitat for Acartia tonsa (Copepoda) in the Río de la Plata, Argentina-Uruguay. Journal of Sea Research 85: 197-204.

Diaz RJ \& R Rosenberg. 2008. Spreading dead zones and consequences for marine ecosystems. Science 321: 926-929.

Elliott DT, JJ Pierson \& MR Roman. 2013. Predicting the effects of coastal hypoxia on vital rates of the planktonic copepod Acartia tonsa Dana. PLoS ONE 8(5): e63987. <doi:10.1371/journal.pone.0063987>

Escamilla JB, E Suárez-Morales \& R Gasca. 2001. Distribución del zooplancton durante flujos de marea opuestos en el complejo lagunar de Chelem, Yucatán, México. Revista de Biología Tropical 49(1): 47-51.

Escamilla JB, U Ordóñez-López \& E Suárez-Morales. 2011. Variabilidad espacial y estacional de Acartia (Copepoda) en una laguna costera del sur del Golfo de México. Revista de Biología Marina y Oceanografía 46: 379-390.

Gasca R \& I Castellanos. 1993. Zooplancton de la Bahía de Chetumal, Mar Caribe, México. Revista de Biología Tropical 41:619-625.

Gasca R, I Castellanos \& E Suárez-Morales. 1994. Análisis preliminar y perspectivas de una comunidad ecológica fronteriza: El zooplancton de la Bahía de Chetumal. En: Suárez-Morales E (ed). Estudio integral de la frontera México-Belice. Tomo IV: Recursos Naturales, pp. 119-142. Centro de Investigaciones de Quintana Roo (CIQRO), México.

González JG \& TE Bowman. 1965. Planktonic copepods from Bahía Fosforescente, Puerto Rico, and adjacent waters. Proceedings of the U.S. National Museum 117: 241-304.

Herrera-Silveira J, JA Arreola-Lizárraga \& J Ramírez. 2009. Cambios hidrológicos y de estado trófico entre los años 2000 y 2006. En: Espinoza-Avalos J, GA Islebe \& HA Hernández-Arana (eds). El sistema ecológico de la bahía de Chetumal / Corozal: costa occidental del Mar Caribe, pp. 21-27. ECOSUR, Chetumal.
Isaza CFA, PC Sierra-Correa, M Bernal-Velásquez, LM Londoño \& W Troncoso. 2006. Global International Waters Assessment Caribbean Sea/Colombia \& Venezuela, Central America \& Mexico, GIWA Regional assessment, 96 pp. University of Kalmar on behalf of United Nations Environment Programme, Nairobi.

Legendre L \& P Legendre. 1983. Numerical ecology. Developments in environmental modelling 3, 419 pp. Elsevier Scientific, Amsterdam.

Lopes RM, R do Vale \& FP Brandini. 1998. Composição, abundância e distribuição espacial do zooplâncton no complexo estuarino de Paranaguá durante o inverno de 1993 e o verão de 1994. Revista Brasileira de Oceanografía 46: 195-211.

López BP, G Aceves \& I Castellanos. 2005. Distribución y abundancia del zooplancton del complejo lagunar ChacahuaLa Pastoría, Oaxaca, México. Revista Mexicana de Biodiversidad 76: 63-70.

Morales-Vela B, D Olivera-Gómez, JE Reynolds III \& GB Rathbun. 2000. Distribution and habitat use by manatees (Trichechus manatus manatus) in Belize and Chetumal Bay, Mexico. Biological Conservation 95: 67-75.

Ordóñez-López U \& M Ornelas-Roa. 2003. Variaciones de la comunidad de copépodos pláncticos en el gradiente estuarino-costero de Celestún, Yucatán, México. Hidrobiológica 13: 231-238.

Ortíz-Hernández MC \& R Sáenz-Morales. 1999. Effects of organic material and distribution of fecal coliforms in Chetumal Bay, Quintana Roo, Mexico. Environmental Monitoring and Assessment 55: 423-434.

Poulet SA \& R Williams. 1991. Characteristics and properties of copepods affecting the recruitment of fish larvae. Bulletin of the Plankton Society of Japan, Special Volume 1991: 271-290.

Shannon CE. 1948. A mathematical theory of communication. Bell System Technical Journal 27: 379-423, 623-656.

Sokal RR \& FJ Rohlf. 1995. Biometry, 887 pp. WH Freeman, New York.

Sterza JM \& L Loureiro-Fernandes. 2006. Zooplankton community of the Vitória bay estuarine system (Southeastern Brazil) characterization during a three-year study. Brazilian Journal of Oceanography 54: 95-105.

Suárez-Morales E. 1994. Copépodos pláncticos de la Bahía de Chetumal, México (1990-1991). Caribbean Journal of Science 30: 181-188.

Suárez-Morales E. 1995. Clave ilustrada para la identificación de los copépodos pláncticos de la Bahía de Chetumal. AvaCient 12: 16-24.

Suárez-Morales E. 2003. Bibliografía comentada y perspectivas en el estudio de los copépodos pelágicos del Golfo de México y Zonas adyacentes del Atlántico Noroccidental. En: Barreiro T, ME Meave, M Signoret \& G Figueroa (eds). Planctología mexicana, pp. 143-156. Sociedad Mexicana de Planctología AC/ UAM-Iztapalapa/ ECOSUR, México. 
Suárez-Morales E \& R Gasca. 1996. Planktonic copepods of Bahía de la Ascensión, Caribbean coast of Mexico: a seasonal survey. Crustaceana 69: 162-174.

Suárez-Morales E, MO Zamponi \& R Gasca. 1997. Hydromedusae (Cnidaria: Hydrozoa) of Bahía de la Ascensión, Caribbean coast of Mexico: a seasonal survey. Proceedings of the 6th International Conference on Coelenterate Biology, National Natuurhistorisch Museum, Leiden, pp. 465-472.

Teixeira PF, S Kaminski, TR Avila, AP Cardozo, JGF Bersano \& A Bianchini. 2010. Diet influence on egg production of the copepod Acartia tonsa (Dana, 1896). Anais da Academia Brasileira de Ciências 82: 333-339.

Tester P \& JT Turner. 1988. Comparative carbon-specific ingestion rates of phytoplankton by Acartia tonsa, Centropages velificatus and Eucalanus pileatus grazing on natural phytoplankton assemblages in the plume of the Mississippi River (northern Gulf of Mexico continental shelf). Hydrobiologia 167/168: 211-217.
Tester P \& JT Turner. 1991. Why is Acartia tonsa restricted to estuarine habitat? Bulletin of the Plankton Society of Japan, Special Volume 1991: 603-611.

Turner JT. 1984. Zooplankton feeding ecology: contents of fecal pellets of the copepods Temora turbinata and T. stylifera from continental shelf and slope waters near the mouth of the Mississippi River. Marine Biology 82: 73-83.

Turner JT \& P Tester. 1989. Zooplankton feeding ecology: nonselective grazing by the copepods Acartia tonsa Dana, Centropages velificatus De Oliveira, and Eucalanus pileatus Giesbrecht in the plume of the Mississippi River. Journal of Experimental Marine Biology and Ecology 126: $21-43$.

Vásquez-Yeomans L, I Castellanos, E Suárez-Morales \& R Gasca. 2012. Variación espacio-temporal de la biomasa de zooplancton en un sistema estuarino del Caribe Occidental durante dos ciclos anuales. Revista de Biología Marina Oceanografía 47: 213-225. 